\title{
ANÁLISE DOS BENEFÍCIOS RELACIONAIS OBSERVADOS POR USUÁRIOS DE SERVIÇOS
}

ANALYSIS OF RELATIONAL BENEFITS IN THE SERVICES CONTEXT

\section{MÁRCIO DE OLIVEIRA MOTA}

Mestre pela Universidade Estadual do Ceará (Uece).

Professor assistente da Faculdade Integrada do Ceará (FIC).

Rua Eduardo Sabóia, 600, ap. 1.302 - Fortaleza - CE - CEP 60175-145

E-mail:marcio@marciomota.com

ANA AUGUSTA FERREIRA FREITAS

Doutora pela Universidade Federal de Santa Catarina (UFSC)

Professora titular da Universidade Estadual do Ceará (Uece). Rua 8 de Setembro, 1.130, ap. 1604 - Fortaleza - CE - CEP 60175-120 E-mail: freitas8@terra.com.br 


\section{RESUMO}

Este estudo tem como objetivo analisar as relações entre os benefícios relacionais percebidos por consumidores e seus antecedentes e conseqüentes no contexto de serviços. O estudo compreendeu uma pesquisa descritiva, do tipo survey, de natureza quantitativa. A pesquisa de campo foi realizada com 4I5 consumidores que informaram o tipo de serviço analisado. Os dados coletados foram submetidos a testes estatísticos por meio da técnica de modelagem em equações estruturais, no intuito de testar o modelo proposto. Ressalte-se o ineditismo do modelo proposto, pois, neste estudo, foi feita uma avaliação da relação dos benefícios relacionais e seus antecedentes e conseqüentes. Este estudo concluiu que os benefícios relacionais são percebidos pelos consumidores independentemente do grau de customização dos serviços ou da intensidade de contato. Foram encontradas significativas associações entre satisfação com os empregados e os demais construtos, portanto pôde-se inferir a importância do elo entre empregados e consumidores.

\section{PALAVRAS-CHAVE}

Serviços; Marketing de relacionamento; Benefícios relacionais; Satisfação; Fidelidade.

\section{ABSTRACT}

This study aims to analyze the relationship among relational benefits and their antecedent and consequent variables in the services field. A quantitative descriptive research was conducted. The field research was carried out with 415 consumers who pointed out their types of services. The collected data were analyzed by statistical tests, including structural equation modeling given by statistical tests and models based on previous studies. This study presents an original model because it evaluates for the first time relational benefits and their antecedent and consequent variables together. The outcomes of the proposed model concluded that relational 
benefits are perceived by costumers either in high contact customized providers or moderate contact non-personalized providers. Furthermore, it seems that there are strong associations among satisfaction with employees and the other constructs, valuing the importance of relational chains between employees and costumers.

\section{KEYWORDS}

Services; Relationship marketing; Relational benefits; Satisfaction; Loyalty.

\section{INTRODUÇ ÃO}

De acordo com diversas pesquisas empíricas, clientes fiéis geram benefícios às empresas, mas esses aspectos da construção dos relacionamentos levaram ao questionamento dos benefícios que o marketing de relacionamento oferecia aos consumidores, a outra parte, pouco estudada no processo. Surgiam nesse momento os chamados benefícios relacionais, cerne deste estudo, que são divididos por Gwinner et al. (I998) em benefícios de confiança, benefícios sociais e benefícios de tratamento especial. Embora esse estudo pioneiro seja datado de há quase dez anos, observou-se que há uma escassez de estudos, em especial empíricos, sobre benefícios relacionais dentro de uma perspectiva avaliativa desses benefícios para os clientes, como conseqüência da fidelidade a provedores de serviços. Portanto, estudos sobre benefícios relacionais têm como foco averiguar se existem benefícios percebidos dentro de um relacionamento com orientação ao consumidor.

Há também uma lacuna no desenvolvimento de construto único no escopo do marketing de relacionamento, que são os benefícios relacionais, uma vez que Gummesson $(2002,2005)$ menciona que relacionamentos de longo prazo são lucrativos para todas as partes envolvidas, inclusive para os consumidores. Assim, a questão principal para a qual se busca resposta por meio deste estudo está contida na seguinte questão: Quais os fatores responsáveis pela formação da percepção de benefícios relacionais no contexto de serviços, e quais as conseqüências dessa percepção?

Essa pergunta origina o objetivo principal do estudo, que é analisar as relações entre os benefícios relacionais percebidos por consumidores e seus antecedentes e conseqüentes no contexto de serviços. Especificamente, pretende-se I. verificar a influência das características de serviços nos benefícios relacionais, 2. examinar a influência do envolvimento pessoal nos benefícios relacionais perce- 
bidos pelos consumidores, e 3. verificar a influência dos benefícios relacionais percebidos no comprometimento e na satisfação, recomendação e fidelidade às empresas e aos empregados.

A estrutura do artigo foi construída de forma que sintetize os principais elementos da teoria e suas interfaces com a literatura de benefícios relacionais, que se segue de um modelo empírico original e das considerações finais, que apontam implicações teóricas e gerenciais advindas da pesquisa.

\section{BENEFÍ́CIOS RELACIONAIS}

Há quase vinte e cinco anos, Berry (I983) conceituou marketing de relacionamento, trazendo uma nova abordagem à teoria do marketing, como parte de diversos artigos e livros sobre marketing e comportamento do consumidor, em especial com enfoques na perspectiva gerencial (SHETH; MITTAL, 2004). Como conseqüência disso, doze anos depois, Berry (I995) afirmou que o conceito de marketing de relacionamento estava no estágio de maturidade, uma vez que as pesquisas empíricas registravam que clientes fiéis geravam benefícios às empresas (PETERSON, I995), destacando-se, entre outros, aumento de receita (REICHHELD, I996a, I996b, 2002), previsibilidade de vendas e lucratividade (AAKER, I992), aquisição de mais itens na loja (REICHHELD, I996a, I996b; BERRY, I996) e recomendações via boca a boca (BUTLER, I998; HARRISONWALKER, 200I; ZEITHAML; BITNER, 2003; REICHHELD, 2003; CHETOCHINE, 2006; MCCONELL; HUBA, 2006, 2007).

Por um lado, os relacionamentos de longo prazo geram resultados positivos às empresas e aos consumidores como atestam diversos estudos sobre o assunto. Por outro lado, há poucos estudos teóricos e empíricos sobre os benefícios aos clientes fiéis a provedores de serviços (BENDAPUDI; BERRY, I997; HENNIG-THURAU; HANSEN, 2006). Dentre eles, notadamente, citam-se os trabalhos desenvolvidos nos Estados Unidos (GWINNER et al., I998; REYNOLDS; BEATTY, I999a; HENNIG-THURAU et al., 2002; KINARD; CAPELLA, 2006), na Ásia, com pesquisas na Tailândia e em Taiwan, respectivamente (PATTERSON; SMITH, 200I; YEN; GWINNER, 2003), na Europa (HENNIG-THURAU; HANSEN, 2006) e no Brasil (GONÇALVES FILHO; ELIAS; LEITE, 2006).

Os estudos empíricos sobre relacionamentos propuseram modelos explicativos do sucesso ou da falha dos relacionamentos entre os provedores de serviços e consumidores, enquanto os estudos sobre benefícios relacionais procuram averiguar se existem benefícios percebidos no relacionamento com o consumidor. Nesse caso, a qualidade do relacionamento pressupõe que a fidelidade dos consumidores é baseada em poucos construtos, os quais refletem o grau de 
adequação do relacionamento pela perspectiva do consumidor (HENNIG-THURAU; KLEE, I997).

Benefícios aos consumidores se baseiam na informação de que eles escolhem produtos e/ou serviços pelos benefícios que desejam (HALEY, I968; GUTMAN, I982). Esses benefícios podem ser produtos, aquisição de informação ou interação social (DARDEN; DORSCH, I990). Além disso, os consumidores podem receber benefícios de relacionamentos interpessoais por meio de familiares, amigos, vendedores, empregados ou provedores de serviços (BEATTY et al., I996; BITNER I995; GWINNER et al., I998) e manter esses relacionamentos com vendedores/ empregados para satisfazer certos desejos e necessidades por meio de benefícios provindos desses relacionamentos (REYNOLDS; BEATTY, I999a).

A pesquisa pioneira de Gwinner et al. (I998) aponta que os consumidores que têm relacionamentos com provedores de serviços não querem apenas a entrega satisfatória do serviço em si, mas também querem receber desses relacionamentos benefícios adicionais como laços sociais, confiança e de tratamentos especiais. O benefício de confiança é considerado o mais importante benefício relacional (cf. GWINNER et al., I998). Para Hennig-Thurau et al. (2002), esse benefício se refere às percepções dos consumidores acerca da redução de ansiedade e do aumento do conforto em saber o que se espera do encontro do serviço. Além disso, ele aumenta a confiança percebida no provedor de serviços e diminui a percepção do risco (BERRY, I995; BITNER, I995). Gwinner et al. (I998) corroboram as idéias de Sheth e Parvatiyar (I995), afirmando que os consumidores preferem reduzir as opções, e, nesse caso, os relacionamentos de longo prazo ajudam a reduzir riscos por meio dos benefícios de confiança.

Gwinner et al. (I998) apontam que os benefícios sociais ficariam em segundo lugar, no nível de importância, entre os benefícios relacionais, e HennigThurau et al. (2002, p. 234) explicam que esses benefícios são

ligados à parte emocional do relacionamento e caracterizados pelo reconhecimento pessoal dos consumidores aos empregados, à familiaridade dos consumidores com os empregados e à criação e ao desenvolvimento de amizades entre consumidores e empregados.

Goodwin (I997) e Czepiel (I990) afirmam que muitos consumidores recebem benefícios sociais por terem desenvolvido um relacionamento com os provedores de serviços, e esses benefícios aparecem com mais freqüência nos serviços em que há maior grau de contato interpessoal entre consumidores e empregados. Gwinner et al. (I998) consideram os benefícios de tratamentos especiais menos importantes, e tanto esses autores como Reynolds e Beatty (i999b) argumentam 
que esses benefícios acontecem no âmbito econômico ou de forma customizada. Para Hennig-Thurau et al. (2002, p. 234), os benefícios ocorrem "quando os consumidores relacionais recebem preços mais baixos, serviços mais rápidos ou serviços adicionais personalizados".

Os antecedentes dos benefícios relacionais foram pioneiramente identificados por Kinard e Capella (2006) e consistem nas características dos serviços e do envolvimento pessoal. De acordo com Lovelock (I983), os serviços estão longe de ser classificados como homogêneos, e essa heterogeneidade diz respeito a todos os provedores e ofertas de serviços, já que os consumidores podem perceber a diferença na qualidade dos serviços ofertados, dependendo do desempenho da entrega. Czepiel (I990) afirma que, por causa dessas diferenças, os provedores de serviços tendem a personalizá-los de tal modo que atendam às necessidades dos consumidores. Nesse sentido, Kinard e Capella (2006) verificaram que os serviços com maior grau de interação face a face entre empresas e consumidores têm maior probabilidade de ser percebidos pelos consumidores como benefícios relacionais. Ressalte-se que os serviços relacionais podem variar por tipo de serviço, por causa do elevado nível de contato interpessoal e de elevada customização dos serviços, e em situações de baixo contato e de serviços padronizados. Por sua vez, o envolvimento do consumidor é influenciado por características do produto, por características pessoais relacionadas do consumidor e por fatores situacionais do processo de decisão de compra (cf. KINARD; CAPELLA, 2006). Zaichkowsky (I994, p. 342) definiu o construto envolvimento como "uma relevância percebida de uma pessoa a um objeto baseado nas necessidades inerentes, valores e interesses".

Os conseqüentes dos benefícios relacionais são a satisfação com empregados e empresas, a confiança com empregados e empresas, a fidelidade com empregados e empresas, a recomendação boca a boca com empregados e empresas e o comprometimento, cujos detalhes são descritos a seguir. Crosby e Stephens (I987) verificaram que a satisfação em serviços é evidenciada por meio das experiências em três vertentes distintas: a pessoa de contato ou o empregado, o serviço em si e o provedor de serviços. Swan e Oliver (I989) verificaram que os consumidores têm diferentes expectativas quando interagem com diferentes objetos e sujeitos, em especial quando interagem com as lojas (objetos) e seus empregados (sujeitos). Assim, os consumidores podem experimentar satisfação de forma diferenciada em relação às empresas e nas suas interações com os empregados.

Hennig-Thurau et al. (2006) postulam que a satisfação do cliente com o empregado está associada à satisfação com a empresa e enfatizam os motivos pelos quais algumas empresas conseguem ou não construir e manter relacionamentos com os consumidores, indicando que a satisfação com os empregados é um fator de forte influência à fidelidade aos empregados. Beatty et al. (1996) observaram 
que os sentimentos positivos dos consumidores em relação aos empregados são transferidos às empresas, corroborando as idéias de Crosby et al. (I990).

Por causa da intangibilidade dos serviços, Gremler e Brown (I999) e Gremler et al. (200I) afirmam que os consumidores de serviços são especialmente inclinados a confiar em fontes pessoais, e, como conseqüência, essa confiança impacta na recompra e na intenção de compra, pelo fato de a recomendação boca a boca influenciar o processo de decisão de compra (BUTLER, I998; REICHHELD, 2006). Isso significa que a ligação entre transmissão da recomendação do remetente e lucratividade das empresas é mediada por diversas variáveis relacionadas às respostas psicológicas e comportamentais das pessoas. Reichheld e Sasser (I990) e Singh (I990) indicam que um elevado nível de satisfação resulta em recomendação boca a boca positiva, conseqüentemente os consumidores fazem boas referências sobre empresas e empregados (CROSBY et al., I990). A recomendação boca a boca é geralmente um ato de transferência informal de uma informação entre consumidores em relação às compras e ao consumo. Ela pode ser negativa ou positiva, representando, quase sempre, uma variável dependente final, ou seja, um construto conseqüente. Alguns estudos indicam que a qualidade percebida e o valor percebido no serviço são associados positivamente com a recomendação boca a boca (DANAHER; RUST, I996; FULLERTON; TAYLOR, 2002).

Finalmente, o comprometimento é um dos construtos de grande importância para o marketing de relacionamento (cf. GARBARINO; JOHNSON, I999). Moorman et al. (I992, p. 316) definiram-no como "um desejo contínuo em manter um valioso relacionamento"; Morgan e Hunt (i994, p. 23) entendem-no como "uma crença de troca entre partes em que esse relacionamento existente é tão importante como garantir máximos esforços para mantê-lo". Em síntese, é possível afirmar que os relacionamentos, em especial na área de serviços, são construídos nos fundamentos do comprometimento mútuo (cf. BERRY, I996) e que as partes identificam o comprometimento entre trocas como elementos-chave para alcançar melhores resultados entre si, mantendo assim esse valioso atributo.

\section{CONSTRUÇÃO DO MODELO E PROCEDIMENTOS METODOLÓGICOS}

Para atingir o objetivo proposto por este estudo, uma pesquisa descritiva foi realizada com o objetivo de testar hipóteses específicas e examinar relações entre variáveis. Com base nos modelos de Reynolds e Beatty (I999b), Hennig-Thurau et al. (2002) e Kinard e Capella (2006), observou-se a possibilidade de propor um novo modelo (Figura I) para avaliar quais os fatores responsáveis pela formação da percepção de benefícios relacionais, no contexto de serviços, e quais as conseqüências dessa percepção. 


\section{FIGURA I}

\section{MODELO PROPOSTO DOS BENEFÍCIOS RELACIONAIS E SEUS ANTECEDENTES E CONSEQU゚ENTES}

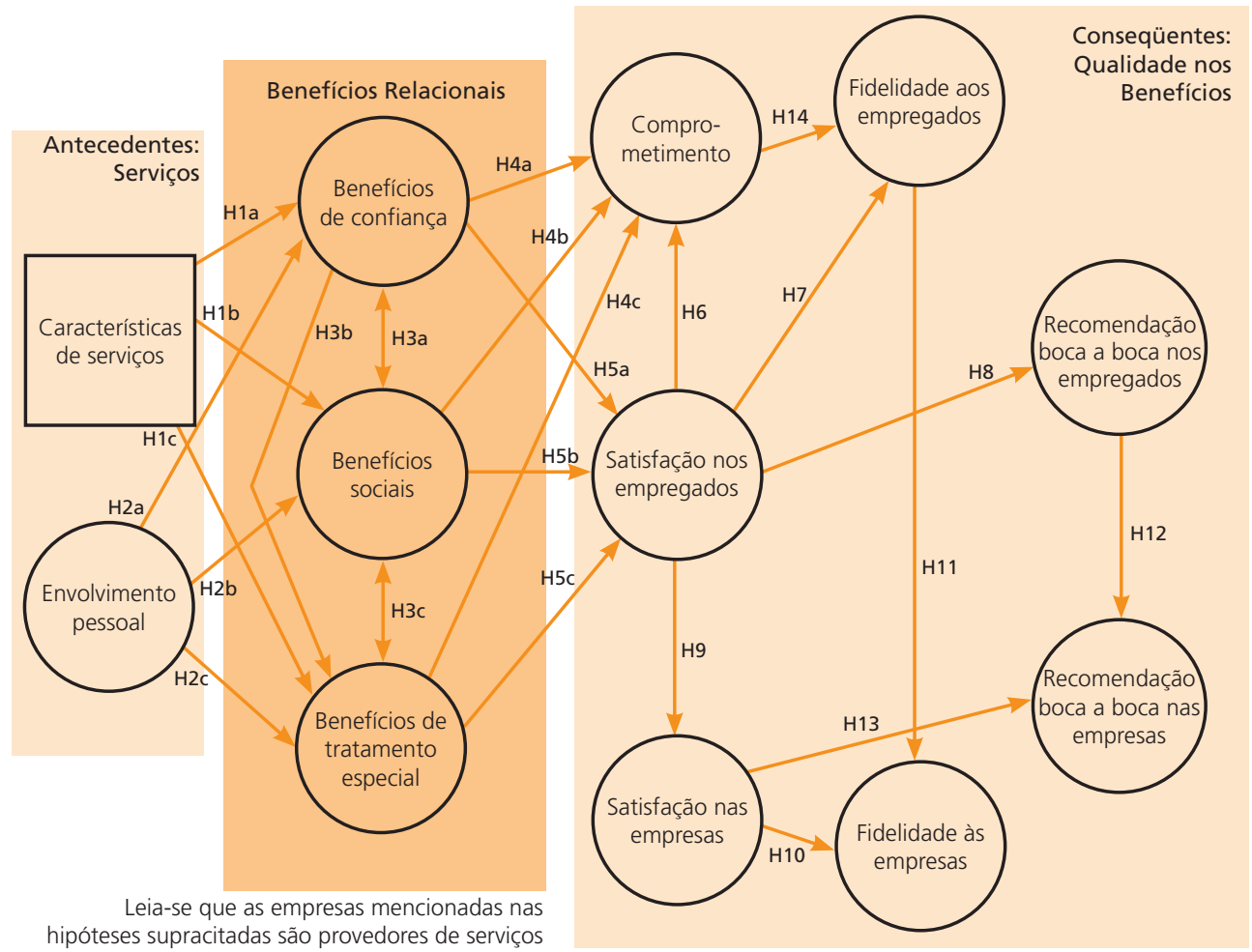

A definição da população que foi amostrada, ante os objetivos e a definição do problema de pesquisa proposto por este estudo, consistiu no total da população de todos os consumidores de serviços, classificando os usuários por nível de contato e tipo de provedor de serviços. Em relação ao tipo de contato, os usuários de serviços poderiam ser classificados em três categorias: alto contato, contato moderado e baixo contato. Em relação ao tipo de serviço oferecido pelo provedor, a classificação utilizada foi serviços customizados, semicustomizados e serviços padronizados. O tipo de amostragem usado nesta pesquisa foi a amostra nãoprobabilística por conveniência, uma vez que a pesquisa foi realizada em salas de aula, com a devida autorização dos docentes, com estudantes universitários de diversos cursos e instituições de ensino superior, públicas e particulares, nos três turnos (manhã, tarde e noite). As hipóteses a serem verificadas no modelo proposto são as seguintes: 
- Hi: provedores de serviços que oferecem mais (menos) serviços customizados são mais (menos) prováveis de influenciar a percepção pelos consumidores dos benefícios relacionais e seus conseqüentes.

- H2: o elevado (baixo) envolvimento de consumidores é mais (menos) provável de ter benefícios relacionais percebidos em provedores de serviços.

- H3: os benefícios relacionais sofrem influência mútua entre si.

- $\mathrm{H}_{4}$ : o comprometimento é influenciado positivamente pelos benefícios relacionais.

- H5: a satisfação com os empregados é influenciada positivamente pelos benefícios relacionais.

- H6: o comprometimento é influenciado positivamente pela satisfação dos clientes com os empregados.

- H7: a fidelidade aos empregados é influenciada positivamente pela satisfação dos consumidores com eles.

- H8: a recomendação boca a boca dos empregados é influenciada positivamente pela satisfação dos consumidores com os empregados.

- Hy: a satisfação com os provedores de serviços é influenciada positivamente pela satisfação com os empregados.

- Hıo: a fidelidade aos provedores de serviços é influenciada positivamente pela satisfação com os provedores de serviços.

- Hıs: a fidelidade aos provedores de serviços é influenciada positivamente pela fidelidade aos empregados.

- Hı2: a recomendação boca a boca dos provedores de serviços é influenciada positivamente pela recomendação boca a boca dos empregados.

- Hiz: a recomendação boca a boca dos provedores de serviços é influenciada positivamente pela satisfação com os provedores de serviços.

- Hi4: a fidelidade aos empregados é influenciada positivamente pelo comprometimento.

É importante ressaltar que as escalas de mensuração adotadas nesta pesquisa foram retiradas dos três modelos revisados na teoria (REYNOLDS; BEATTY, I999b; HENNIG-THURAU et al., 2002; KINARD; CAPELLA, 2006) e estão devidamente validadas e testadas pelo estudo desses autores, apresentando resultados consistentes tanto em termos de validade interna (o modo como foi definido o plano amostral mediu aquilo que se queria medir, isolando as verdadeiras relações causais) quanto externa (as verdadeiras relações causais identificadas podem ser aplicadas em outras circunstâncias semelhantes e generalizadas para todo o universo).

Salienta-se que, como as medidas utilizadas neste trabalho foram retiradas de diferentes fontes (REYNOLDS; BEATTY, I999b; HENNIG-THURAU et al., 2002; 
KINARD; CAPELLA, 2006) e são originárias de estudos norte-americanos, elas foram traduzidas para a língua portuguesa por meio da técnica de tradução reversa (DILLON et al., I993) pelos autores deste estudo e validadas por dois lingüistas, a fim de garantir a manutenção do significado e da precisão das medidas.

A escala do instrumento de coleta de dados foi pré-testada em vinte usuários de provedores de três tipos de serviços durante o mês de julho de 2007. Os resultados do pré-teste indicaram que nenhuma alteração na escala foi sugerida. Contudo, ocorreram algumas indicações de alterações de frases para melhor compreensão dos respondentes.

Quando a pesquisa se propõe a utilizar a análise de dados multivariada, é necessário realizar uma verificação preliminar dos dados de tal modo que possa identificar valores omissos e observações substancialmente diferentes das demais (outliers) (KLINE, I998; BYRNE, 200I). Essa análise foi feita seguindo as sugestões de Kline (I998), Byrne (200I), Tabachnick e Fidell (200I), Malhotra (200I) e Hair et al. (2005) para os testes de normalidade, linearidade, homocedasticidade e multicolinearidade dos dados.

Conforme Hair et al. (2005), a modelagem de equações estruturais (MEE) é uma técnica multivariada que combina aspectos da regressão linear múltipla e análise fatorial, a fim de estimar simultaneamente uma série de relações dependentes e inter-relacionadas. Dessa forma, este estudo seguiu os procedimentos indicados por esses autores. Inicialmente, os modelos de mensuração foram examinados e somente após o estabelecimento da validade e confiabilidade das medidas utilizadas é que foi realizado o exame do modelo estrutural, isto é, o modelo que inclui as relações entre construtos foi gerado. Essa abordagem se chama two-step approach e é sugerida por Anderson e Gerbing (I988) e Schumacker e Lomax (I996).

Para a estimação do modelo proposto, inicialmente foram construídos modelos de mensuração para cada construto, nos quais foram especificadas as relações entre a variável latente (elemento não-observável) e os itens das escalas (elementos observáveis). Essas relações foram analisadas por meio da análise fatorial confirmatória, construto a construto, avaliando a confiabilidade e a validade convergente por meio dos procedimentos de Fornell e Larker (I98I) e de Garver e Mentzer (I999).

Em seguida, construiu-se um modelo de mensuração geral com todas as variáveis latentes e todas as variáveis observadas. Nessa etapa, analisou-se o ajuste geral do modelo. Salienta-se que, enquanto o modelo de mensuração relaciona os construtos às medidas, o modelo de estimação, baseado no modelo empírico apresentado na Figura I, relaciona um construto a outro (cf. JARVIS et al., 2003). O software utilizado para a estimação do modelo foi o Amos 7.0 que a faz por meio da utilização da técnica de estimação da máxima verossimilhança. 
Informa-se ainda que, embora Tabachnick e Fidell (200I) e Hair et al. (2005) afirmem que os valores aceitáveis dos índices de ajustes estatísticos em modelos de equações estruturais devam ser superiores a 0,90, Cote (200I) indica que, quando há um número grande de relações e construtos, pode ocorrer degradação do ajuste e aponta que, para modelos complexos, como é o caso do modelo proposto neste artigo, valores acima de o,80 estão dentro dos limites aceitáveis.

- Realizou-se o teste de hipóteses para cada caminho estrutural do diagrama dos caminhos (path diagram) do modelo estrutural para identificar se os valores dos t-values são superiores a I,96 para $\mathrm{p}<0,05$ ou a I,33 para $\mathrm{p}<0,0 \mathrm{I}$.

- Verificou-se se a carga fatorial de cada item no construto é de no mínimo 0,50, se o cálculo do alfa de Cronbach é de no mínimo o,70 e se a variância extraída é de no mínimo o,50 (cf. HAIR et al., 2005).

Para avaliar se as co-variantes, categoria de serviços padronizados e customizados, têm ou não impacto nas relações entre os construtos do modelo, foi utilizada a análise multigrupo na modelagem de equações estruturais. Nessa técnica, a base de dados é dividida em grupos de acordo com as variáveis categóricas, e os modelos são estimados simultaneamente (BYRNE, 200I). A hipótese nula testada é que os dados de cada grupo pertencem à mesma população, ou seja, os grupos não diferem e as covariantes não impactam nas relações estruturais.

\section{ANÁLISE E DISCUSSÃO dOS RESULTADOS}

Dentre os 4I5 usuários pesquisados, 55\% eram homens, 45\% mulheres; $80 \%$ tinham idade entre I7 e 25 anos; e $49 \%$ indicaram manter uma relação periódica e constante com o tipo de serviço customizado e de alto contato, $7 \%$ em serviços semicustomizados e de contato moderado e $44 \%$ de serviços padronizados e de contato moderado. Foi utilizada a técnica estatística de análise fatorial confirmatória, com o intuito de fazer a validação dos construtos, cujo objetivo foi avaliar se os conjuntos de indicadores utilizados estavam medindo o que, teoricamente, deveriam medir, apresentando validade convergente e discriminante, e também se eram consistentes apenas para os construtos para os quais foram desenvolvidos, apresentando unidimensionalidade. Apenas os construtos envolvimento, benefícios de confiança e de tratamentos especiais apresentaram problemas com a unidimensionalidade, por isso houve necessidade de reespecificações nesses construtos. 
Após a verificação individual dos construtos, o modelo de mensuração como um todo foi avaliado por meio de índices de ajuste do modelo, da validade discriminante e da multicolinearidade. Para que as reespecificações feitas na etapa de validação dos construtos fossem avaliadas, foi estimado um modelo de mensuração com as respectivas reespecificações com todos os construtos na forma original, indicadores incluídos e sem nenhuma covariância adicionada, apontando que o modelo reespecificado é o mais ajustado, confirmando as reespecifações feitas.

Verificou-se também a validade discriminante por meio da comparação da variância extraída de cada construto com suas variâncias compartilhadas, e, em seguida, foi realizada a avaliação da multicolinearidade pelas intercorrelações entre os construtos. Para a estimação do modelo estrutural, as correlações entre os construtos do modelo de mensuração são substituídas pelos caminhos hipotetizados no modelo teórico. Assim, o modelo estrutural estimado é apresentado por meio dos índices de ajuste na Tabela I.

A medida de ajustamento qui-quadrado/gl é satisfatória. O RMSEA apresentou um índice levemente superior a 0,08 , apontado por Byrne (200I) como uma adequação mediana, e os índices NFI, IFI, TLI e CFI, que comparam o modelo estrutural proposto ao modelo nulo, estão dentro ou muito próximos do satisfatório, e, conforme sugestão de Cote (200I), um patamar de o,80 é um bom índice para modelos complexos. Nesse caso, o estudo em questão é complexo, pois possui diversas relações entre um elevado número de variáveis. Segundo Cote (200I), é preferível aceitar o modelo com esses índices de ajuste a reespecificálo sem razões teóricas fortes para tanto. O modelo está no limiar do satisfatório. De qualquer forma, um modelo rival foi estimado para avaliar uma alternativa ao modelo proposto. Contudo, os índices de ajustamento apresentaram-se mais baixos que os indicados pelo modelo estrutural na Tabela I.

\section{TABELA I}

ÍNDICES DE AJUSTE DO MODELO ESTRUTURAL

\begin{tabular}{cccccccccc}
\hline Modelo & $X^{2}$ & $p$ & $g l$ & $X^{2} / g l$ & NFI & IFI & TLI & CFI & RMSEA \\
\hline Estrutural & 3357,648 & 0,000 & 879 & 3,820 & 0,782 & 0,829 & 0,815 & 0,828 & 0,083 \\
\hline
\end{tabular}

Observação: selecionaram-se as seguintes medidas e parâmetros de avaliação, segundo indicações de Cote (200I): $\chi^{2} / g l$ menor que 5; RMSEA para valores entre 0,05 e 0,08 são satisfatórios. Para os índices NFI, IFI, TLI e CFI, valores acima de o,80 são satisfatórios.

Ressalte-se que as demais relações foram todas estatisticamente significativas, dentre as quais se identifica: a satisfação dos clientes com os funcionários 
(SatisfFunc) e com os provedores de serviços (SatisfPS), a recomendação boca a boca positiva dos provedores de serviços (BocaPS) e a fidelidade aos provedores de serviços (LealPS). Foram também indicados os valores do $\mathrm{R}^{2}$ para cada variável dependente para que fosse observada melhor sua explicação nas relações entre os caminhos explicitados na Tabela 2.

A influência das co-variantes, categorias de serviços padronizados e customizados, foi testada por meio da análise multigrupo. Para o teste da hipótese Hi, para as co-variantes características dos serviços e benefícios relacionais, o modelo com parâmetros livres foi estimado. Os resultados mostraram que, para os serviços customizados, houve o mesmo número de hipóteses rejeitadas, porém apenas as hipóteses $\mathrm{H}_{5}$ c e Hio foram rejeitadas mutuamente.

\section{TABELA 2}

TESTES DE HIPÓTESES DO MODELO ESTRUTURAL

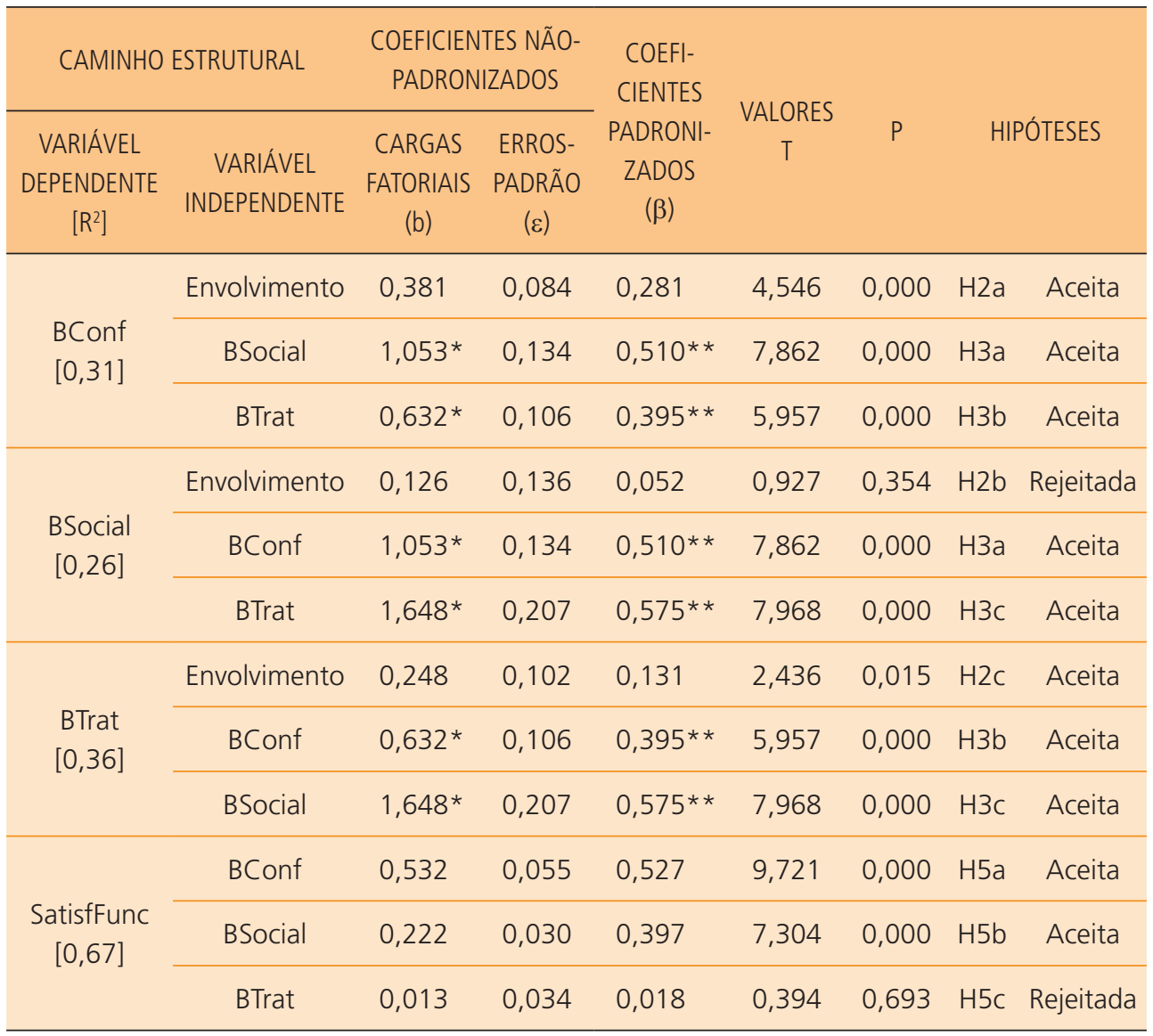




\section{TABELA 2 (CONTINUAÇÃO)}

\section{TESTES DE HIPÓTESES DO MODELO ESTRUTURAL}

\begin{tabular}{|c|c|c|c|c|c|c|c|c|}
\hline CAMINHO & ESTRUTURAL & $\begin{array}{l}\text { COEFICIEN } \\
\text { PADRON }\end{array}$ & $\begin{array}{l}\text { TES NÃO- } \\
\text { IZADOS }\end{array}$ & $\begin{array}{l}\text { COEFI- } \\
\text { CIENTES }\end{array}$ & & & & \\
\hline VARIÁVEL & & CARGAS & ERROS- & PADRONI- & $T$ & $P$ & & ÓTESES \\
\hline $\begin{array}{c}\text { SatisfPS } \\
{[0,82]}\end{array}$ & SatisfFunc & 0,979 & 0,057 & 0,905 & 17,275 & 0,000 & H9 & Aceita \\
\hline $\begin{array}{c}\text { BocaFunc } \\
{[0,48]}\end{array}$ & SatisfFunc & 0,935 & 0,074 & 0,694 & 12,704 & 0,000 & $\mathrm{H} 8$ & Aceita \\
\hline BocaPS & BocaFunc & 0,475 & 0,051 & 0,475 & 9,309 & 0,000 & $\mathrm{H} 12$ & Aceita \\
\hline$[0,64]$ & SatisfPS & 0,511 & 0,061 & 0,410 & 8,371 & 0,000 & $\mathrm{H} 13$ & Aceita \\
\hline LealFunc & SatisfFunc & 0,477 & 0,071 & 0,367 & 6,733 & 0,000 & $\mathrm{H} 7$ & Aceita \\
\hline$[0,70$ & Compr & 0,576 & 0,058 & 0,570 & 9,871 & 0,000 & $\mathrm{H} 14$ & Aceita \\
\hline LealPS & SatisfPS & 0,049 & 0,069 & 0,614 & 0,705 & 0,481 & $\mathrm{H} 10$ & Rejeitada \\
\hline$[0,77]$ & LealFunc & 0,877 & 0,079 & 0,854 & 11,047 & 0,000 & $\mathrm{H} 11$ & Aceita \\
\hline & SatisfFunc & 0,254 & 0,107 & 0,198 & 2,367 & 0,018 & $\mathrm{H} 6$ & Aceita \\
\hline Compr & BConf & 0,225 & 0,094 & 0,174 & 2,395 & 0,017 & $\mathrm{H} 4 \mathrm{a}$ & Aceita \\
\hline$[0,49]$ & BSocial & 0,112 & 0,049 & 0,155 & 2,274 & 0,023 & $\mathrm{H} 4 \mathrm{~b}$ & Aceita \\
\hline & BTrat & 0,304 & 0,054 & 0,328 & 5,683 & 0,000 & $\mathrm{H} 4 \mathrm{C}$ & Aceita \\
\hline
\end{tabular}

*Covariância. e ***arrelação. Valores de t $>$ I,96 $\rightarrow$ p $<0,05$ e de $t>2,33 \rightarrow p<0,0$ I.

Embora o número de hipóteses rejeitadas para os serviços customizados tenha sido o mesmo para os serviços padronizados (cinco hipóteses), o último obteve também todas as três hipóteses rejeitadas (H2b, $\mathrm{H}_{5} \mathrm{c}$ e Hro) em relação ao modelo geral com 4I5 casos. Pode-se afirmar que o modelo geral proposto explica melhor a influência dos benefícios relacionais em seus conseqüentes, independentemente da dimensão de serviços. De qualquer forma, foi necessário verificar se as diferenças são estatisticamente significativas e o modelo com parâmetros fixos foi estimado. Isso foi feito com a denominação, caminho a caminho, dos parâmetros por letras iguais nos dois submodelos (serviços customizados e 
padronizados). Assim, fez-se a comparação entre o qui-quadrado do modelo livre com graus de liberdade e do modelo restrito com graus de liberdade. A diferença do qui-quadrado para a diferença dos graus de liberdade não foi significativa. Logo, as diferenças encontradas entre os grupos não são estatisticamente significativas, e a característica de serviços não está exercendo influência sobre os caminhos estimados. Dessa forma, a hipótese Hi não foi suportada pelos dados.

Com base nos dados das hipóteses $\mathrm{H} 2 \mathrm{a}, \mathrm{H} 2 \mathrm{~b}$ e H2c, observa-se que os resultados dessa hipótese corroboram os estudos de Kinard e Capella (2006), segundo os quais os benefícios de confiança são os que melhor respondem quando há um maior envolvimento pessoal, e ratificam também o trabalho de Patterson e Smith (200I), quando perceberam o fator cultural na Tailândia, explicando uma maior importância dos benefícios de tratamento especial em vez dos benefícios sociais.

Os resultados encontrados da hipótese $\mathrm{H}_{3}$ foram semelhantes ao modelo de Reynolds e Beatty (I999b), uma vez que eles também observaram uma elevada correlação entre os benefícios relacionais. Ressalta-se apenas que esses autores se utilizaram apenas de dois benefícios relacionais (benefícios sociais e benefícios funcionais que incluem os de confiança e de tratamento especial). Por sua vez, Gonçalves Filho, Elias e Leite (2006) obtiveram resultados que mostraram que não havia significância entre os benefícios sociais e de confiança, e que havia uma correlação negativa entre os benefícios de tratamento especial e de confiança. A análise comparativa entre os resultados do presente estudo e os de Gonçalves Filho, Elias e Leite (2006) dá notoriedade ao fato de que culturas diferentes podem ser divergentes quando se trata de benefícios relacionais, fato ocorrido na Tailândia (PATTERSON; SMITH, 200I).

Os resultados deste estudo divergem apenas na hipótese H4a em relação aos estudos realizados por Gonçalves Filho, Elias e Leite (2006) e Hennig-Thurau et al. (2002), pois nesses dois últimos não houve influência significativa no comprometimento pelo benefício de confiança. Por sua vez, Morgan e Hunt (I994) afirmam que a confiança é fundamental na relação de compromisso entre os atores em questão. Finalmente, ressalte-se que os benefícios de tratamento especial foram os que mais influenciaram o comprometimento no estudo $\mathrm{H}_{3}$, anteriormente apresentado. Há nos entrevistados também a relação de que quanto mais benefícios extras recebem, maior será o seu comprometimento com a empresa, todavia deve-se ter cuidado com os tratamentos especiais, pois, de acordo com Hennig-Thurau et al. (2002), recompensas levam apenas a um comportamento temporário de fidelidade.

Com os resultados da $\mathrm{H}_{5}$ corroboram-se os estudos de Hennig-Thurau et al. (2002) e os de Gonçalves Filho, Elias e Leite (2006), implicando que o benefício que mais influencia a satisfação com os empregados é o benefício de confiança. 
O resultado sobre a relação positiva entre os benefícios sociais e a satisfação com os empregados foi também encontrado no estudo de Reynolds e Beatty (I999b), quando esses autores verificaram que quanto maiores os benefícios relacionais percebidos pelos consumidores, maior é a satisfação com os empregados. Quanto à H6, o resultado ratifica os estudos de Garbarino e Johnson (I999), quando estes afirmam que a satisfação é antecedente do comprometimento com impacto positivo e significativo, e com os estudos de Hennig-Thurau et al. (2002) e Gonçalves Filho, Elias e Leite (2006), implicando que a satisfação também exerce poder de mediadora entre os benefícios relacionais e o comprometimento.

Para responder à $\mathrm{H} 7$, esse resultado apresentou valores muito próximos dos encontrados no trabalho de Hennig-Thurau et al. (2002) quando a satisfação de uma forma geral impacta com a fidelidade dos consumidores também de uma forma geral. Por sua vez, o estudo de Reynolds e Beatty (I999b), com a avaliação isolada entre a fidelidade com empregados e a satisfação dos consumidores também com empregados, apresentou impacto positivo e significativo.

Em relação à H8, é importante ressaltar dois aspectos fundamentais: a) o impacto entre essas duas variáveis é o terceiro maior encontrado no modelo proposto b) e, no estudo de Gonçalves Filho, Elias e Leite (2006), verificou-se a relação de maior impacto do modelo. Ademais, tanto nos modelos de Hennig-Thurau et al. (2002) como no de Reynolds e Beatty (I999b), houve impactos fortes e significativos. Dessa forma, pode-se inferir que a satisfação dos consumidores com os empregados está no modelo proposto como a única variável antecessora da variável recomendação boca a boca de empregados e respondeu fortemente nessa relação, corroborando os estudos de Howard e Sheth (I969), Reichheld e Sasser (I990) e Singh (I990) de que a satisfação resulta em boca a boca positiva.

Para responder à $\mathrm{H}_{9}$, o presente estudo seguiu a proposta de Reynolds e Beatty (I999b) de dividir a satisfação em duas partes, sendo a primeira a satisfação com os empregados e a segunda a satisfação com as empresas, no caso em questão, nos provedores de serviços. Ressalte-se que esse impacto é o maior valor obtido no modelo proposto, ratificando os prévios estudos de Beatty et al. (I996) e Crosby et al. (I990), os quais observaram que respostas positivas de satisfação com os empregados de uma empresa são transmitidas diretamente à empresa.

Com respeito à Hıo e Hıı, mais uma vez o presente estudo seguiu a sugestão de Reynolds e Beatty (I999b) quando dividiu a fidelidade em duas ramificações: nos empregados e nas empresas. De acordo com Macintosh e Lockshin (I997), a fidelidade a empregados de uma empresa é antecedente da própria empresa, pois os sentimentos em relação aos empregados são passados às empresas (BEATTY et al., I996). Esses resultados mostram que os consumidores se sentem fiéis a uma empresa quando também se sentem fiéis aos empregados dela, tornando essa última seu único antecessor. 
Quanto à Hı2 e Hı3, o presente estudo seguiu novamente a sugestão de Reynolds e Beatty (I999b) quando dividiu a recomendação boca a boca em duas ramificações: nos empregados e nas empresas. Ressalte-se que os dois antecessores (satisfação com as empresas e recomendação boca a boca aos empregados) da recomendação boca a boca dos provedores de serviços influenciaram positivamente com significativos impactos. Pode-se afirmar então que, quando se recomendam os empregados da empresa, o consumidor também tende a recomendar positivamente as empresas, e, quando o consumidor está satisfeito com as empresas, este também tende a recomendar positivamente as empresas a terceiros.

Por último, quanto à Hi4, observa-se que a relação do modelo proposto foi também testada nos estudos de Gonçalves Filho, Elias e Leite (2006) e de HennigThurau et al. (2002), os quais obtiveram valores positivos e significativos. Ressalte-se que, originalmente, essa relação foi retirada dos estudos de Garbarino e Johnson (I999), que obtiveram resultados também positivos e significativos. Esses últimos autores postulam que o comprometimento influencia na continuidade de relações futuras em relações de trocas.

\section{CONSIDERAÇÕES FINAIS}

Este estudo verificou que o grau de percepção dos benefícios relacionais percebidos depende dos tipos de serviços prestados e varia entre customizados e de alto contato a serviços padronizados e de contato moderado. Verificou-se que não houve distinção na percepção dos benefícios relacionais e seus conseqüentes. Esse resultado permite entender que mesmo as empresas que não oferecem serviços customizados ou de maior contato com os consumidores devem estar preocupadas com os benefícios ofertados a eles, pois resultarão em conseqüências de marketing essenciais à empresa, como satisfação, comprometimento, fidelidade e recomendação boca a boca positiva.

Verificou-se também que há influência positiva entre os benefícios sociais e de tratamento especial, ou seja, os consumidores que percebem que ganham algum tratamento especial, como descontos extras ou atendimento prioritário, associam um vínculo social atribuído a esses tratamentos. É importante também salientar que elevados impactos dos benefícios relacionais no comprometimento podem ser relatados como efeito de fidelidade temporária por parte dos consumidores aos provedores de serviços, ou seja, os consumidores serão fiéis durante o tempo em que o prestador de serviço oferecer tratamentos especiais, vantagens percebidas, especificamente as financeiras. No momento em que seus concorrentes oferecerem maiores vantagens financeiras, os consumidores terão maior 
possibilidade de migrar, no intuito de obter ainda mais vantagens. Por isso, os prestadores de serviços devem tomar muito cuidado quando oferecem primordialmente os benefícios de tratamentos especiais em vez de atribuírem mais valor aos outros dois tipos de benefícios relacionais.

Há um construto fundamental que serviu como mediador principal entre os benefícios relacionais e seus conseqüentes: a satisfação com os empregados. As influências entre satisfação com os empregados e os demais construtos são inferidas à importância do elo entre empregados e consumidores e à extrema relevância de qualidade no relacionamento, em especial em provedores de serviços. É possível afirmar que os sentimentos positivos que os consumidores têm com os empregados são traduzidos em sentimentos também positivos às empresas das quais esses empregados fazem parte. Este estudo proporcionou outra perspectiva da relação entre satisfação, fidelidade e recomendação boca a boca, à medida que esses três construtos foram divididos em satisfação, fidelidade e recomendação positiva nos empregados e nos provedores de serviços. Essa divisão proporcionou uma observação sobre as relações diretas e significativas entre as influências dos sentimentos atribuídos aos empregados e nos provedores de serviços.

Observa-se também a importância dos empregados na recomendação boca a boca das empresas como um dos principais meios de comunicação e propagação de serviços de uma empresa e como uma forma de avaliá-los por meio das recomendações. A satisfação com os empregados e, conseqüentemente, a satisfação com os provedores de serviços e a recomendação boca a boca dos empregados foram fundamentais para a recomendação boca a boca positiva aos provedores de serviços. Portanto, mais uma vez afirma-se que os provedores de serviços devem entender os empregados como o meio mais seguro para proporcionar relações mais fortes e duradouras com seus consumidores. As empresas que possuem um programa freqüente de treinamento com seus empregados devem mensurar os riscos de deserção e, caso seja detectado um médio ou alto risco, oferecer um plano concreto de carreiras e de incentivos salariais.

As empresas prestadoras de serviços devem ainda quantificar os benefícios relacionais e informá-los, de forma direta, aos seus consumidores, pois muitos deles não têm idéia de tais benefícios. Assim, eles perceberão e compreenderão o significado dessa relação e seus ganhos diretos e indiretos. Essa percepção pode levar os consumidores a refletir no momento de uma eventual ruptura com seu atual prestador de serviços ou até mesmo a uma comparação imediata com os outros provedores de serviços com os quais eles já tenham tido relações, facilitando assim a simplificação na escolha do melhor serviço e a diminuição do risco na escolha e no recebimento do serviço per se.

Sugere-se entender mais profundamente como a relação entre os consumidores e os empregados pode representar riscos para a empresa, pois a elevada 
rotatividade de empregados ou a ida destes aos concorrentes pode enfraquecer essa relação e/ou iniciar um processo migratório de consumidores/clientes aos concorrentes. Sob a perspectiva acadêmica, este trabalho, além de contribuir com a introdução de um modelo original de percepção de benefícios relacionais no contexto de serviços no Brasil, agrega ao corpo de conhecimento da área de marketing a relação entre seus antecedentes e conseqüentes sob um prisma transversal.

\section{REFERÊNCIAS}

AAKER, D. A. The value of brand equity. Journal of Business Strategy, v.13, p. 27-32, Jul./Aug. I992.

ANDERSON, J. C.; GERBING, D. W. Structural equation modeling in practice: a review and recommended two-step approach. Psychological Bulletin, v. I03, p. 4II-423, I988.

BEATTY, S. et al. Customer-sales associate retail relationships. Journal of Retailing, v. 72, n. 3, p. 223-247, 1996.

BENDAPUDI, N.; BERRY, L. L. Customers' motivations for maintaining relationships with service providers. Journal of Retailing, v. 73, n. I, p. 15-37, I997.

BERRY, L. L. Emerging perspectives on services marketing. American Marketing Association, Chicago, p. $25-28,1983$.

. Relationship marketing of services: growing interest, emerging perspectives. Journal of the Academy of Marketing Science, v. 23, n. 4, p. 236-245, I995.

. Serviços de satisfação máxima: guia prático de ação. Tradução On Great Service. Rio de Janeiro: Elsevier, I996.

BITNER, M. J. Building service relationships: it's all about promises. Journal of the Academy of Marketing Science, v. 23, p. 246-5I, Fall I995.

BUTLER, F. A. Word of mouth: understanding and managing referral marketing. Journal of Strategic Marketing, v. 6, p. 24I-254, I998.

BYRNE, B. M. Structural equation modeling with AMOS: basic concepts, applications and programming. New Jersey: Lawrence Erlbaum Associates, 200I.

CHETOCHINE, G. Buzz marketing: sua marca na boca do cliente. Tradução Arlete Simille Marques. São Paulo: Financial Times-Prentice Hall, 2006.

COTE, J. Structural equations modeling: improving model fit by correlating errors. Journal of Consumer Psychology, v. Io, n. 2, p. 83-100, 200 I.

CROSBY, L. A.; STEPHENS, N. Effects of relationship marketing on satisfaction, retention, and prices in the life insurance industry. Journal of Marketing Research, v. 24, p. 404-4II, Nov. I987. CROSBY, L. A. et al. Relationship quality in services selling: an interpersonal influence perspective. Journal of Marketing, v. 54, p. 68-8I, Jul. I990.

CZEPIEL, J. A. Service encounters and service relationships: implications for research. Journal of Business Research, v. 20, n. I, p. I3-2I, I990. 
DANAHER, P. J.; RUST, R. T. Indirect financial benefits from service quality. Quality Management Journal, v. 3, n. 2, p. 63-75, I996.

DARDEN, W. R.; DORSCH, M. J. An action strategy approach to examining shopping behavior. Journal of Business Research, v. 2I, n. 3, p. 289-308, Nov. I990.

DILLON, W. R. et al. Research in a marketing environment. St. Louis: Times Mirror, I993.

FORNELL, C.; LARCKER, D. Evaluating structural equation models with unobservable variables and measurement error. Journal of Marketing Research, v. I8, p. 39-50, Feb. I98I.

FULLERTON, G.; TAYLOR, S. Mediating, interactive, and non-linear effects in service quality and satisfaction research. Canadian Journal of Administrative Sciences, v. I9, n. 2, p. I24-136, 2002.

GARBARINO, E.; JOHNSON, M. The different roles of satisfaction, trust and commitment for relational and transactional consumers. Journal of Marketing, v. 63, n. 2, p. 70-87, Apr. I999.

GARVER, M. S.; MENTZER, J. T. Logistics research methods: employing structural equation modeling to test for construct validity. Journal of Business Logistics, v. 20, n. I, p. 33-57, I999.

GONÇALVES FILHO, C.; ELIAS, C. L.; LEITE, R. S. Antecedentes da lealdade de clientes: um estudo empírico no varejo. Revista de Negócios, Blumenau, v. II, n. I, p. 2-I7, jan./mar. 2006.

GOODWIN, C. Communality as a dimension of service relationships. Journal of Consumer Psychology, v. 5, p. 387-415, I997.

GREMLER, D. D.; BROWN, S. W. The loyalty ripple effect: appreciating the full value of customers. International Journal of Service Industry Management, v. IO, n. 3, p. 27I-29I, I999.

GREMLER, D. et al. Generating positive word-of-mouth communication through customer-employee relationships. International Journal of Service Industry Management, v. I2, n. I, p. 44-59, 200 I.

GUMMESSON, E. Relationship marketing in the new economy. Journal of Relationship Marketing, v. I, n. I, p. 37-58, 2002.

Marketing de relacionamento total: gerenciamento de marketing, estratégias de relacionamento e abordagem de CRM para economias de rede. Tradução Marina Barbieri Campomar e Jonathan Hogan. 2. ed. Porto Alegre: Bookman, 2005.

GUTMAN, J. A means-end chain model based on consumer categorization processes. Journal of Marketing, v. 46, n. 2, p. 60-72, 1982.

GWINNER, K. P. et al. Relational benefits in services industries: the customer's perspective. Journal of the Academy of Marketing Science, v. 26, n. 2, p. IOI-II4, I998.

HAIR, J. et al. Análise multivariada de dados. Tradução Adonai Schlup Sant'Anna e Anselmo Chaves Neto. 5. ed. Porto Alegre: Bookman, 2005.

HALEY, R. I. Benefit segmentation: a decision-oriented research tool. Journal of Marketing, v. 32, n. 3, p. 30-35, Jul. I968.

HARRISON-WALKER, L J. The measurement of word-of-mouth communication and an investigation of service quality and customer commitment as potential antecedents. Journal of Service Research, v. 4, n. I, p. 60-75, Aug. 200I.

HENNIG-THURAU, T.; HANSEN, U. Relationship marketing - some reflections on the state-ofthe-art of the relational concept. In: (Ed.). Relationship marketing: competitive advantage through customer satisfaction and customer retention. Berlin, New York: Springer, 2006. HENNIG-THURAU, T.; KLEE, A. The impact of customer satisfaction and relationship quality on customer retention: a critical reassessment and model development. Psychology \& Marketing, v. I4, p. 737-765, Dec. I997. 
HENNIG-THURAU, T. et al. Understanding relationship marketing outcomes: integration of relational benefits and relationship quality. Journal of Service Research, v. 4, n. 3, p. 230-247, 2002.

Why customers build relationships with companies - and why not. In: (Ed.). Relationship marketing: competitive advantage through customer satisfaction and customer retention. Berlin, New York: Springer, 2006.

HOWARD, J. R.; SHETH, J. N. The theory of buyer behavior. New York: John Wiley and Sons, I969.

JARVIS, C. B. et al. A critical review of construct indicators and measurement model misspecification in marketing and consumer research. Journal of Consumer Research, v. 30, p. 199-2I8, Sept. 2003.

KINARD, B. R.; CAPELLA, M. L. Relationship marketing: the influence of consumer involvement on perceived service benefits. Journal of Services Marketing, v. 20, n. 6, p. 359-368, 2006.

KLINE, R. B. Principles and practice of structural equation modeling. New York: The Guilford Press, I998.

LOVELOCK, C. H. Classifying services to gain strategic marketing insights. Journal of Marketing, v. 47 , n. 3, p. 9-20, Summer I983.

MACINTOSH, G.; LOCKSHIN, L. S. Retail relationships and store loyalty: a multi perspective. International Journal of Research in Marketing, v. I4, n. 5, p. 487-497, I997.

MALHOTRA, N. K. Pesquisa de marketing: uma orientação aplicada. Tradução Nivaldo Montingelli Jr. e Alfredo Alves de Farias. Porto Alegre: Bookman, 200I.

MCCONELL, B.; HUBA, J. Buzzmarketing: criando clientes evangelistas. Tradução: R. Brian Taylor. São Paulo: MBooks de Brasil, 2006.

Citizen marketers: when people are the message. Chicago: Kaplan Publishing, 2007.

MOORMAN, C. et al. Relationships between providers and users of market research: the dynamics of trust within and between organizations. Journal of Marketing Research, v. 29, p. 314-328, Aug. I992.

MORGAN, R. M.; HUNT, S. D. The commitment-trust theory of relationship marketing. Journal of Marketing, v. 58, n. 3, p. 20-38, Jul. I994.

PATTERSON, P. G.; SMITH, T. Relationship benefits in service industries: a replication in a Southeast Asian context. Journal of Services Marketing, v. I5, n. 6, p. 425-443, 200 I.

PETERSON, R. A. Relationship marketing and the consumer. Journal of the Academy of Marketing Science, v. 23, n. 4, p. 278-28I, Fall I995.

REICHHELD, F. F. Loyaty-based management. Harvard Business Review, v. 7I, p. 64-73, Mar./Apr. I996а.

A estratégia da lealdade. Tradução: Ana Beatriz Rodrigues e Priscilla Martins Celeste. Rio de Janeiro: Campus, I996b.

Princípios de lealdade: como os líderes atuais constroem relacionamentos duradouros e lucrativos. Tradução: Cristiana Serra. Rio de Janeiro: Campus, 2002.

The number one you need to grow. Harvard Business Review, v. 8I, n. I2, p. 46-54, Nov./ Dec. 2003.

REICHHELD, F. F.; SASSER JR., W. E. Zero defections: quality comes to services. Harvard Business Review, v. 68, n. 5, p. I05-III, Sept./Oct. I990. 
REYNOLDS, K. E.; BEATTY; S. E. Customer benefits and company consequences of customersalesperson relationships in retailing. Journal of Retailing, v. 75, p. II-32, Spring I999a.

A relationship customer typology. Journal of Retailing, v. 75, p. 509-523, Winter I999b.

SCHUMACKER, R. E.; LOMAX, R. G. A beginner's guide to structural equation modeling. New Jersey: Lawrence Erlbaum Associates, I996.

SHETH, J.; MITTAL, B. Customer behavior: a managerial perspective. 2. ed. Ohio: South Western Thomson Learning, 2004.

SHETH, J.; PARVATYAR, A. Relationship marketing in consumer markets: antecedents and consequences. Journal of the Academy of Marketing Science, v. 23, n. 4, p. 255-27I, I995.

SINGH, J. Voice, exit and negative word-of-mouth behaviors: an investigation across three service categories. Journal of The Academy of Marketing Science, v. I8, p. I-I5, Winter 1990.

SWAN, J. E.; OLIVER, R. L. Postpurchase communications by consumers. Journal of Retailing, v. 65, n. 4, p. 516-533, I989.

TABACHNICK, B. G.; FIDELL, L. S. Using multivariate statistics. 4. ed. Needham Heights, MA: Allyn and Bacon, 200I.

YEN, H. J. R.; GWINNER, K. P. Internet retail customer loyalty: the mediating role of relational benefits. International Journal of Service Industry Management, v. I4, n. 5, p. 483-500, 2003.

ZAICHKOWSKY, J. L. The personal involvement inventory: reduction, revision and application to advertising. Journal of Advertising, v. 23, p. 59-70, Dec. I994.

ZEITHAML, V. A.; BITNER, M. J. Marketing de serviços. Tradução: Martin Albert Haag e Carlos Alberto Silveira Netto Soares. Porto Alegre: Bookman, 2003. 